\title{
$7 \times 149$ Gbit/s PAM4 Transmission over 1 km Multicore Fiber for Short-Reach Optical Interconnects
}

\author{
Oskars Ozolins ${ }^{1}$, Xiaodan Pang ${ }^{2,1}$, Aleksejs Udalcovs ${ }^{1}$, Rui Lin ${ }^{2,4}$, Joris Van Kerrebrouck ${ }^{3}$, Lin Gan ${ }^{4}$, \\ Lu Zhang ${ }^{2}$, Ming Tang ${ }^{4}$, Songnian Fu $^{4}$, Richard Schatz ${ }^{2}$, Urban Westergren ${ }^{2}$, Gunnar Jacobsen ${ }^{1}$, \\ Deming Liu ${ }^{2}$, Weijun Tong, Guy Torfs ${ }^{3}$, Johan Bauwelinck ${ }^{3}$, Jiajia Chen ${ }^{2}$, Sergei Popov ${ }^{2}$, and Xin Yin ${ }^{3}$ \\ ${ }^{I}$ Networking and Transmission Laboratory, RISE Acreo AB, Kista, Sweden \\ ${ }^{2}$ KTH Royal Institute of Technology, Kista, Sweden \\ ${ }^{3}$ IDLab, INTEC, Ghent University - imec, Gent, Belgium \\ ${ }^{4}$ Huazhong University of Science and Technology, Wuhan, China \\ ${ }^{5}$ Yangtze Optical fiber and Cable Joint Stock Limited Company, Wuhan, China \\ Author e-mail address: oskars.ozolins@ri.se
}

\begin{abstract}
We transmit $80 \mathrm{Gbaud} / \lambda$ /core PAM4 signal enabled by $1.55 \mu \mathrm{m}$ EML over $1 \mathrm{~km} 7$-core fiber. The solution achieves single-wavelength and single-fiber $1.04 \mathrm{Tbit} / \mathrm{s}$ post-FEC transmission enhancing bandwidth-density for short-reach optical interconnects.

OCIS codes: (200.4650) Optical interconnects; (060.2360) Fiber optics links and subsystems
\end{abstract}

\section{Introduction}

Intra-datacenter interconnects have been experiencing a tremendous growth of capacity to enable web-based highperformance applications [1-3]. High capacity demand from datacenter applications has become the technology driver for high bandwidth (BW) short-reach communications [4]. Earlier demonstrations, e.g. [5-7], include twolanes $400 \mathrm{GbE}$ client-side links interconnecting the high bandwidth systems and providing a smaller footprint while consuming less power. Pulse amplitude modulation with four levels (PAM4) is the modulation format of choice for physical layer specifications of $400 \mathrm{GbE}$ interfaces [3]. Line side optics need to scale on faster pace to support tremendous bandwidth growth. Therefore, digital signal processing enhancements are critical factors for the optical devices to enable higher linearity, extinction ratio, and tighter integration with electronics [1]. Space-division multiplexing (SDM) used today in datacenters is in the form of parallel single mode fibers thanks to less dominant fiber cost for short-reach links [8]. Meanwhile, SDM fibers are actively explored to scale up system capacity through spatial efficiency [2]. A combination of high bandwidth devices and spatial efficiency through multicore fibers (MCFs) seems to be a viable solution to enable high bandwidth-density, having enormous potential to increase the throughput per single-wavelength and single-fiber to $1 \mathrm{~Tb} / \mathrm{s}$ for short-reach optical interconnects $[4,9,10]$.

In this paper, we demonstrate $80 \mathrm{Gbaud} / \lambda /$ core PAM4 signal transmission using a monolithically integrated externally modulated laser (EML). We achieve below 7\% overhead $(\mathrm{OH})$ hard-decision forward error correction (HD-FEC) performance over $1 \mathrm{~km}$ single mode 7-core MCF with a negligible inter-core crosstalk and a low-loss fan-in/fan-out device. The solution enables terabit scale $(7 \times 149 \mathrm{Gbit} / \mathrm{s})$ post-FEC transmission speed over $1 \mathrm{~km}$ single-wavelength and single-fiber, greatly enhancing bandwidth-density for short-reach optical interconnects.

\section{Experiment setup}

Figure 1(a) shows the experimental setup for signal generation, transmission and direct detection and includes the 7core fiber cross section as inset. The 80 Gbaud PAM4 signal is generated offline using a $2^{15}-1$ long pseudorandom binary sequence. Then it is up-sampled and filtered with a root-raised-cosine filter having 0.15 roll-off factor. Frequency domain channel pre-equalization is applied for channel frequency response calibration (up to $46 \mathrm{GHz}$ due to the limitation of the arbitrary waveform generator (AWG)). The calibration is based on the end-to-end system measurement as shown in Fig. 1 (b). After pre-equalization 80 Gbaud PAM4 signal is loaded to a 92 GSa/s AWG. Then it is amplified in a $65 \mathrm{GHz}$ electrical amplifier with $11 \mathrm{~dB}$ gain. A monolithically integrated $1.55 \mu \mathrm{m}$ EML (3 dB BW >100 GHz [11]) is used to generate optical the PAM4 signal.
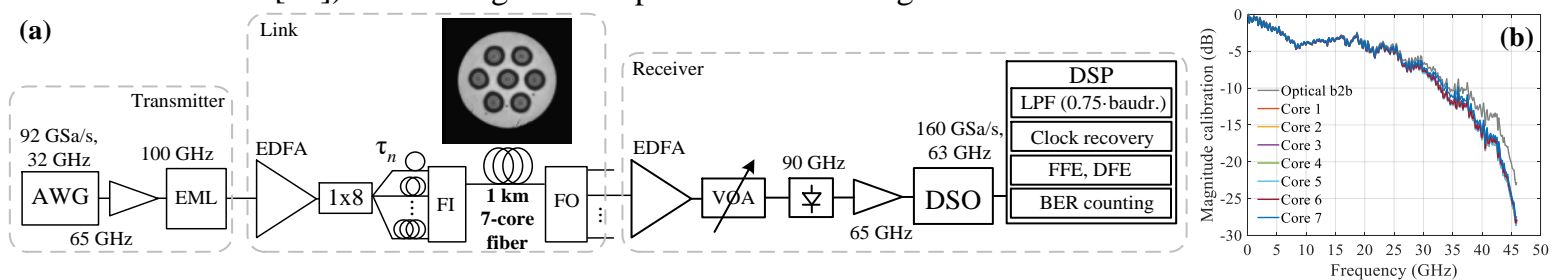

Fig. 1. Experimental setup (a), 7-core fiber cross section (b), channel magnitude calibration frequency response (c) 

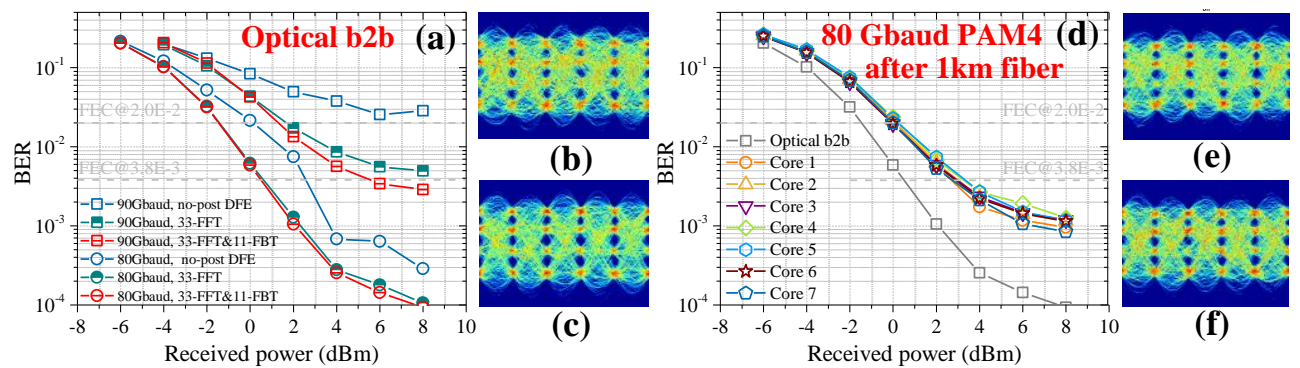

Fig. 2. BER curves for 80 Gbaud and 90 Gbaud PAM4 signal for b2b with different post-equalization (a), 90 Gbaud PAM4 eye diagram with 33 FFT\&11-FBT DFE (b), 80 Gbaud PAM4 eye diagram with 33-FFT\&11-FBT DFE (c), BER curves for 80 Gbaud PAM4 signal for b2b and after $1 \mathrm{~km}$ single mode 7-core MCF transmission (d), 80 Gbaud PAM4 eye diagram for worst core (e) and best core (f).

The distributed feedback laser drive current is set at $115 \mathrm{~mA}$ and the electroabsorption modulator is biased at minus $1.85 \mathrm{~V}$ for linear regime operation [7]. An erbium doped fiber amplifier (EDFA) is used to amplify the signal before $1 \times 8$ splitter, decorrelation stage and fan-in (FI) device. The signal is transmitted over an uncompensated $1 \mathrm{~km}$ single mode 7-core fiber. The cladding diameter of the hexagonal MCF is $150 \mu \mathrm{m}$ and the core pitch is $42 \mu \mathrm{m}$. The average attenuation per core is less than $0.2 \mathrm{~dB} / \mathrm{km}$ at $1550 \mathrm{~nm}$ and the crosstalk between adjacent cores is $-45 \mathrm{~dB} / 100 \mathrm{~km}$. A fan-out (FO) device is used to couple the signals to single core fibers. A variable optical attenuator (VOA) is used to adjust the optical power after the pre-amplifier and before a PIN photodetector ( $3 \mathrm{~dB} \mathrm{BW}>90 \mathrm{GHz}, \mathrm{R}=0.2 \mathrm{~A} / \mathrm{W}$ ). The signal is captured with a $160 \mathrm{GSa} / \mathrm{s} 63 \mathrm{GHz}$ digital storage oscilloscope (DSO). The sampled signal is processed offline using digital signal processing (DSP) routine consisting of a low-pass filter (LPF), a maximum variance timing recovery, a symbol-spaced feed forward equalizer (FFE) and decision-feedback equalizer (DFE) with different configurations of feed-forward taps (FFT) and feedback taps (FBT), and an error counter.

\section{Results and discussions}

In Fig. 2, we show quantitative and qualitative measures for 80 Gbaud and 90 Gbaud PAM4 signals for optical back to back (b2b) and after $1 \mathrm{~km}$ transmission. Bit error rate (BER) curves captured for optical b2b are shown for both baud rates (see Fig. 2(a)), while for the lower baud rate BER after $1 \mathrm{~km}$ transmission over a 7 -core MCF are given (see Fig. 2(d)). In all cases, different post-equalizer configurations are used. In addition, we show eye diagrams of 90 Gbaud and 80 Gbaud PAM4 signals for b2b in Fig. 2(b)\&(c) and 80 Gbaud PAM4 signal after $1 \mathrm{~km}$ single mode 7-core MCF transmission for the worst and the best core in Fig. 2(e)\&(f) at $8 \mathrm{dBm}$ Rx power. One can observe in Fig. 2(a) that the PAM4 signals are impacted by the limited bandwidth of the AWG. For the 90 Gbaud PAM4 signal, both pre-equalization and post-equalization is necessary to reach just below the BER limit of 3.8e-3 for 7\% OH HDFEC. The baud rate was therefore reduced to 80 Gbaud for transmission experiments since in this case preequalization works better improving the performance sufficiently.

\section{Conclusions}

In this paper, we successfully demonstrate a transmission of $80 \mathrm{Gbaud} / \lambda /$ core PAM4 signal over $1 \mathrm{~km}$ single mode 7-core MCF. Single-wavelength and single-fiber $1.04 \mathrm{Tbit} / \mathrm{s}$ post-FEC transmission has been achieved, having an enormous potential to enhance bandwidth-density for short-reach optical interconnects.

\section{Acknowledgement}

This work is supported by the Swedish Research Council (VR), the Swedish Foundation for Strategic Research (SSF), Göran Gustafsson Foundation, the Swedish ICT-TNG project SCENE, FP7 project MIRAGE (ref.318228), H2020 project WIPE (ref.688572), VINNOVA funded Celtic Plus sub-project C2015/3-5 SENDATE-FICUS, Natural Science Foundation of Guangdong Province and National Natural Science Foundation of China.

\section{References}

[1] R. Urata et al., "Datacenter interconnect and networking: from evolution to holistic revolution," in Proc. OFC 2017, W3G.1.

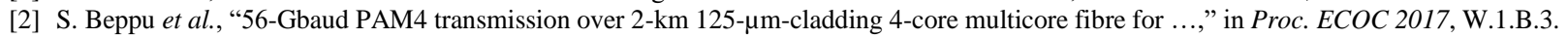

[3] M. Chagnon, D. Plant, "504 and $462 \mathrm{~Gb} / \mathrm{s}$ direct detect transceiver for single carrier short-reach data ...," in Proc. OFC 2017, W3B.2.

[4] D. L. Butler, "Space-division multiplexing (SDM) technology for short-reach fiber optic systems," in Proc. OFC 2016, Tu3I.1.

[5] S. Kanazawa et al., "Transmission of 214-Gbit/s 4-PAM signal using an ultra-broadband lumped- ...," in Proc. OFC 2016, PDP Th5B.3.

[6] S. Lange et al., "100 GBd intensity modulation and direct detection with an InP-based monolithic ...,", in Proc. OFC 2017, PDP Th5C.5.

[7] O. Ozolins et al., "100 Gbaud 4PAM link for high speed optical interconnects," in Proc. ECOC 2017, P2.SC5.6.

[8] P. De Heyn et al., "Ultra-dense 16x56Gb/s NRZ GeSi EAM-PD arrays coupled to multicore fiber for ...," in Proc. OFC 2017, Th1B.7.

[9] T. Hayashi et al., "125- $\mu$ m-cladding 8-core multi-core fiber realizing ultra-high-density cable suitable ...," in Proc. OFC 2015, Th5C.6.

[10]B. Li et al., "Employing multicore fiber in short reach optical networks," in Proc. SUM, (IEEE, 2016), 230-231.

[11] M. Chacinski et al., "ETDM transmitter module for 100-Gb/s Ethernet," PTL 22, 70-72, (2010). 